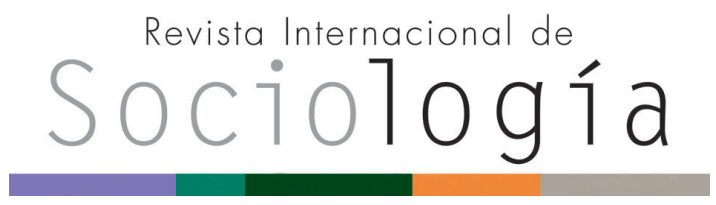

Revista Internacional de Sociología RIS vol. 74 (3), e040, julio-septiembre, 2016, ISSN-L:0034-9712 doi: http://dx.doi.org/10.3989/ris.2016.74.3.040

\section{DEMOCRACIA, ESCALA Y \\ PARTICIPACIÓN. Reflexiones desde las instituciones participativas brasileñas}

\author{
LEONARDO AVRITZER \\ Universidad de Minas Gerais, Brasil. \\ avrizer1@gmail.com
}

\author{
Alfredo Ramos \\ Universidad Complutense de Madrid, España. \\ alfredo@redconvoz.org
}

Cómo citar este artículo / Citation: Avritzer, L. y A. Ramos 2016. "Democracia, escala y participación. Reflexiones desde las instituciones participativas brasileñas". Revista Internacional de Sociología 74(3):e040. doi: http://dx.doi.org/10.3989/ris.2016.74.3.040

\section{Resumen}

Existe un planteamiento generalizado dentro de la teoría democrática, especialmente en la teoría participativa, según el cual, a menor escala (entendida como tamaño del territorio o nivel administrativo), mayores posibilidades de desarrollar procesos participativos de mayor calidad. Para contestar dicha perspectiva, comparamos dos tipos de Instituciones Participativas en Brasil (Consejos y Conferencias Nacionales) en municipios de diferente tamaño y en diferentes niveles administrativos. La hipótesis del texto es que son los niveles intermedios los que presentan una mayor calidad democrática.

\section{Palabras Clave}

Conferencias nacionales; Consejos; Democracia deliberativa; Democracia participativa; Scaling Up.

\section{DEMOCRACY, SCALE AND \\ PARTICIPATION. Reflections from Brazilian participatory institutions}

Copyright: (C) 2016 CSIC. Este es un artículo de acceso abierto distribuido bajo los términos de la licencia Creative Commons Attribution (CC BY) España 3.0.

Recibido: 13/11/2014. Aceptado: 16/09/2015. Publicación online: 22/07/16

\section{Abstract}

A generalized approach in democratic theory exists, especially in participatory theory, in which the smaller the scale (defined as size of territory or administrative level), the more chance there is to develop better quality participatory processes. In response to this perspective, we will compare two types of Participating Institutions in Brazil (Councils and National Conferences) in municipalities of different sizes and administrative levels. The hypothesis of this paper is that intermediate levels are those of higher democratic quality.

\section{KeYWORDS}

Councils; Deliberative Democracy; National Conferences; Participatory Democracy; Scaling Up. 


\section{INTRODUCCIÓN}

La idea de que la participación resulta más fácil, más efectiva y más democrática en territorios con un tamaño más reducido, con un número pequeño de habitantes o en los niveles administrativos más cercanos a la ciudadanía (como las administraciones de proximidad o los gobiernos municipales) forma parte de la tradición de la teoría democrática general y, especialmente, de los estudios sobre la democracia participativa (Przeworski 2010; Dahl 1988; Pateman 1980). Nuestro objetivo es contestar esta afirmación mediante los resultados de una investigación sobre dos tipos de Instituciones Participativas (en adelante IP) en Brasil (Consejos y Conferencias Nacionales). La hipótesis que articula este trabajo es que, en Brasil, si tomamos los tres niveles administrativos (municipal, estatal y federal) y municipios de diferentes dimensiones (capitales, grandes ciudades, medianos y pequeños), hay evidencias para afirmar que el nivel intermedio (el estatal en las Conferencias Nacionales y el de los municipios de medio tamaño en los Consejos) es el que presenta una mejor institucionalidad participativa y una mayor calidad de los procesos participativos.

El primer epígrafe presentará algunas líneas generales sobre cómo se ha abordado teóricamente la relación entre escala (entendida como nivel administrativo y/o tamaño del territorio) y participación. Los dos epígrafes siguientes analizarán Consejos y Conferencias Nacionales en diferentes niveles, para observar la relación entre escala, dinámica institucional de la participación y efectividad de la misma. En las conclusiones se revisará la hipótesis inicial a partir de los datos presentados en los epígrafes anteriores.

Brasil se convirtió en los últimos veinte años en un laboratorio de experiencias de participación y en un marco privilegiado para la discusión sobre las escalas. Desde la redemocratización se ha ido consolidando un panorama diversificado de IP, que son formas diferenciadas de incorporación de los ciudadanos y las asociaciones de la sociedad civil en la deliberación sobre las políticas. Están caracterizadas por: articulación de participación y representación; la transformación de la sociedad civil en una organización política permanente; la interacción con partidos políticos y actores estatales; y, la importancia del diseño institucional para mejorar su efectividad y la interacción entre Estado y sociedad civil, más allá de la mera competición política (Avritzer 2009). Su desarrollo ha tenido diferentes fases. Tras 1989 surgieron los Presupuestos Participativos, a partir de 1990 los Consejos, que se expandieron con la aparición de legislaciones locales a principios de esa década. Por último, en la década del 2000, con la aprobación del Estatuto de la Ciudad, se expanden los Planes Directores. Estas legislaciones ampliaron la presencia de la participación en Brasil más allá de las grandes capitales del Sur y Sureste que, de todas formas, ya no concentraban todas las experiencias, mientras que los Consejos se expandieron hacia ciudades pequeñas y medianas.

Hasta 2003 las principales formas de participación social en Brasil estaban concentradas en el nivel local, donde mostraban importantes efectos (Pires 2011; Avritzer 2010). Sin embargo, existía un importante conjunto de dudas sobre las posibilidades de extender las formas de participación más allá del ámbito municipal (Faria 2005), dudas relacionadas con la escala y la organización del sistema representativo a nivel federal. Este escenario se ha visto modificado con la consolidación de las Conferencias Nacionales y su fuerte impacto en el sistema político (Avritzer y Souza 2013; Progrebinschi y Santos 2010).

Los Consejos son IP donde se comparte el poder de decisión entre representantes de la sociedad civil y actores estatales y su estudio permite investigar la relación entre participación y tamaño de los municipios. Aparecen al inicio de la democratización brasileña y, hoy, más de 10.000 Consejos operan en ciudades brasileñas en la determinación de elementos importantes de diversas políticas públicas como salud y asistencia social.

Las Conferencias Nacionales de Políticas Públicas son una IP de escala nacional (Avritzer y Souza 2013) que nos permite abordar la relación entre participación y diferentes niveles administrativos. Son procesos participativos, deliberativos y representativos que elaboran directrices dirigidas al gobierno nacional y a otros niveles inferiores de gobierno de la federación brasileña (estados y/o municipios). Comienzan con una fase local (en los municipios) donde se eligen propuestas y delegados para la siguiente fase (fase estatal) donde se vuelven a elegir delegados y propuestas para la fase final en Brasilia, donde se reúnen representantes de la sociedad civil y el poder público de todo el país para elaborar las propuestas finales.

Para analizar los Consejos se describirá la estructura y dinámica participativa de 11 municipios pequeños en el estado de Minas Gerais, comparándola con la institucionalidad de 10 municipios de tamaño medio ${ }^{1}$ en dicho estado, analizando los Consejos de Salud, Asistencia Social e Infancia y Adolescencia. El análisis de estos elementos (diseño institucional y dinámica de participación) en las Conferencias Nacionales combinará dos estrategias metodológicas. Por un lado, una encuesta ${ }^{2}$ sobre la ampliación de la participación local al ámbito nacional, para establecer quiénes son los actores participantes de las Conferencias y su relación con la participación local. Por otro lado, una investigación sobre los diseños institucionales de tres Conferencias: Política para Mujeres, Asistencia Social y Control Social y Transparencia. En los tres casos se presentará el ciclo completo del proceso desde la fase inicial en Belo Horizonte a la nacional en Brasilia, pasando por la fase estatal (intermedia entre las dos) de Minas Gerais. 


\section{Algunos PRoblemas teóricos SOBRe PARTICIPACIÓN Y ESCALA}

Desde sus orígenes, la teoría política y democrática ha mostrado su preocupación con la relación entre escala y democracia (Dahl y Tufte 1973). Rousseau (1992) y Montesquieu (1951) insistieron en la relación inversamente proporcional entre el tamaño (extensión del territorio y número de habitantes) y la posibilidad de implicarse en la toma de decisiones, garantizando la voluntad o el interés general. En los debates de The Federalist (1988), la cuestión de la escala se resolvió mediante la innovación que suponía la representación como base de un gobierno republicano. En la primera onda de democratización, que podemos considerar engloba a Francia y los Estados Unidos hasta el inicio del S. XX, el dilema de la escala se traducía en un problema práctico: cuál era la cantidad adecuada de población o de tamaño territorial que permitía una implicación efectiva y equitativa de la ciudadanía en la toma de decisiones en la vida pública. La respuesta siempre señalaba que a mayor población, o mayor extensión, peores posibilidades de participación.

Diferentes teóricos contemporáneos han dado continuidad a estos argumentos. Dahl (1988) recurre a variables como el tamaño, el número de habitantes o el tiempo necesario para hablar en una reunión, señalando que cuanto mayor sean las dos primeras, menores serán las posibilidades de participación o deliberación y mayor la necesidad de delegación. Sartori coincide en afirmar que a mayor tamaño del territorio y número de participantes, menor será la calidad y eficacia de la misma. También varios autores de la teoría participativa afirman que las escalas pequeñas (como los ámbitos comunitarios, la industria, la familia o la educación) son las esferas privilegiadas para la participación. Entre otras razones por basarse en vínculos sociales más sólidos y responder a las necesidades inmediatas de la población (Barber 1984; Pateman 1980).

Sin embargo, estas perspectivas tienen varios problemas. Por un lado presentan una versión limitada de la eficacia de la participación, limitada a problemáticas locales y parciales y consideran la equidad como igual impacto de los participantes en la decisión final. No prestan atención a las condiciones institucionales que posibilitan la participación en condiciones de equidad (entendida como igual oportunidad de participación), o los efectos democratizadores de los diseños institucionales. Por otro lado no consideran la presencia o no de una cultura política más horizontal en el nivel local y en los territorios pequeños. Son precisamente estos los problemas que la participación enfrenta en las escalas pequeñas, tanto en Brasil como en las democracias más avanzadas: el escaso desarrollo institucional al que nos referiremos y la presencia de grupos locales que controlan la política y limitan la participación, fenómeno que podemos denominar "narrowing down».
El segundo problema de la escala es su relación con la complejidad administrativa. La temática de la escala toma nuevos significados al vincularla con la enorme ampliación de las políticas públicas en el territorio que ocurrió a partir del inicio de S. XX (Weber 1976). La especialización en el área política con la determinación burocrática de resultados se convirtió en una concepción consolidada en la política democrática (Bobbio 1987; Sartori 1987). La tensión entre participación y complejidad implicó también que la primera, al vincularse a territorios pequeños o a los niveles inferiores de las administraciones, se centraría en las escalas con menor capacidad de resolución de las demandas de la ciudadanía (Dahl y Tufte 1973), con lo que los procesos de elaboración de demandas quedaban desconectados de las unidades y escalas con mayor capacidad de abordarlas.

La participación se ha reducido a espacios microlocales (barrios, espacios públicos,...) o a la escala de los gobiernos locales, limitándose la capacidad de intervenir y el impacto de estas prácticas, alejadas de los niveles administrativos en los que se toman las decisiones más importantes (Sintomer y Talpin 2011). Gran parte de la teoría participativa ha contribuido a legitimar esta perspectiva añadiendo nuevas cuestiones: la corriente clásica señaló que la participación debía darse vinculada a unos intereses ciudadanos restringidos a los problemas cercanos o a aquellos ámbitos donde su eficacia fuera tangible (Barber 1984; Pateman 1980; MacPherson 1977). Numerosos planteamientos contemporáneos han mantenido esta idea, añadiendo como base el problema epistemológico (Sintomer y Talpin 2011), que considera como virtud de los procesos de participación ciudadana el que permiten incorporar a los ciudadanos a partir de su saber de uso, mejorando la acción de las políticas públicas mediante el conocimiento inmediato del contexto y reduciendo la posibilidad de participar en contextos más complejos o en escalas mayores (Fung y Wright 2003; Smith 2009). Por tanto, pensar la relación entre participación y escala implica ir más allá de la vinculación de la primera al saber de uso y reconocer la diversidad epistémica que estos procesos permiten desplegar (Sintomer 2008).

El resurgir del debate participativo por medio de la ampliación de la deliberación (Habermas 1995; Cohen 1997; Dryzek 2000) colocó el problema de la calidad deliberativa dentro del debate participativo y abrió una posibilidad de solucionar el problema de la escala mediante el tratamiento de cuestiones complejas a nivel local. El problema pasó a ser cómo llevar estas discusiones del nivel local para el nacional o de la sociedad civil al sistema político (Hendriks 2006). Parte de esta problemática se ha intentado abordar desde dos perspectivas. La primera es el giro sistémico de la deliberación, que permite pensar la escala a partir de la interacción de diferentes foros 
en el tiempo, centrándose en la división de tareas entre diferentes espacios (formales e informales) y actores en un sistema de deliberación, la relación entre estos espacios y la pluralidad de repertorios comunicativos (Mansbridge y Parkinson 2012).

La segunda es la perspectiva del scaling up. Levine, Fung y Gastill (2005), señalan dos formas de entender esta cuestión: a) scaling out (aumentar la inclusividad de los procesos, su relación con espacios formales e informales de la esfera pública y la periodicidad de los debates) y; b) scaling up (aumentar los procesos orientados a escalas regionales, nacionales e internacionales, así como el impacto de los mismos). Tres cuestiones se derivan de esta aproximación: inclusión, capilaridad en la esfera pública, e impacto de las decisiones más allá del nivel local.

En la actualidad podemos encontrar dos corrientes dentro del scaling up. Una perspectiva débil con dos posibilidades: a) la deliberación de cuestiones nacionales a nivel local, pero sin que exista conexión o articulación entre estos espacios locales y donde el impacto deja de ser problemático y b) como proceso posdeliberación con escasa institucionalización y referido solo a la adquisición de habilidades o conocimientos que estimulan la participación posterior de la ciudadanía. Dicha participación (en asociaciones voluntarias, campañas electorales..., etc.) sería el mecanismo de relación con las instituciones nacionales (Friedman 2006; Jacobs, Cook y Delli Carpini 2009). La perspectiva fuerte, heredera del giro sistémico, presenta también dos posibilidades. La primera trata de ver la viabilidad de la deliberación como lógica de acción social y política de carácter sostenido. Los problemas aquí tienen que ver con las habilidades, los incentivos y el impacto de la deliberación tanto en la esfera política como en la ciudadana (Bachtiger y Wegmann 2014). La segunda analiza si los productos derivados del trabajo dentro de un minipúblico pueden replicarse dentro de una esfera pública ampliada, centrándose en los modos de incluir, procesar y aceptar demandas dentro del sistema (Niemeyer 2012). Esta perspectiva fuerte amplia la agenda de trabajo del scaling up pero presenta dos límites importantes compartidos con el enfoque sistémico que le sirve de marco de referencia: no presta atención a las instituciones híbridas formadas contemporáneamente por sociedad civil y arena política (como las instituciones participativas) (Avritzer 2009) y se centra únicamente en los minipúblicos sin plantearse las posibilidades de otro tipo de procesos. Un problema similar enfrentan el giro sistémico y el enfoque fuerte del scaling up: la representación, ya que se centran solo en la tipología de representación por similitud de los diseños deliberativos, sin ampliar el enfoque a otras maneras de vincular participación y representación que aparecen en las IP, por ejemplo, y que presentan otras formas de vincular participación y escala.

Tanto las críticas presentadas a los enfoques originales de la relación entre participación y escala, así como los desafíos colocados por la agenda del giro sistémico y el scaling up, nos permiten presentar una agenda de investigación sobre dicha relación que tiene que ver con a) la efectividad de sus diseños institucionales en diferentes escalas; b) la capacidad de inclusión de los mismos; c) su viabilidad, sostenibilidad, transversalidad y grado de consolidación; d) la relación entre diferentes espacios con procesos de participación diferentes; e) la agenda de estas instituciones participativas y la diversidad cognitiva que albergan y/o permiten; f) su vinculación a contextos de complejidad administrativa diferente y su impacto en los mismos y; g) la relación entre participación y representación. A continuación, tras una breve presentación del panorama de las IP en Brasil, y centrándonos en los diseños institucionales de diferentes Consejos y Conferencias Nacionales, abordaremos estos elementos y su relación con la escala. Las condiciones de estos procesos se analizarán considerando municipios de diferente tamaño y el scaling up nacional de la participación como la conexión de fórums de participación que suceden en diferentes lugares, en un periodo de tiempo determinado, para producir debates contemporáneos sobre una misma agenda o temática de políticas públicas.

\section{Consejos y ESCALA EN MUNICIPIOS DE TAMAÑOS DIFERENTES}

El estudio sobre la participación en Brasil ha estado habitualmente más vinculado a las capitales y a las grandes ciudades. Aun cuando sabemos que las experiencias de participación son más comunes en las ciudades de tamaño medio que en las grandes capitales (Wampler 2008) y que estas ciudades cuentan con mayor capacidad tributaria y menor visibilidad en la lucha política nacional, razones que aumentan las posibilidades de poner en marcha experiencias exitosas. Para comparar estas características con las de las pequeñas ciudades estudiamos los casos de 11 municipios de entre 10.000 y 12.000 habitantes y altamente representativos de los pequeños municipios de Brasil, localizados en diferentes regiones del estado de Minas Gerais: Alpinópolis, Alvinópolis, Arinos, Belo Oriente, Bom Sucesso, Campina Verde, Carmo do Cajurú, Carmos do Rio Claro, Chapada do Norte, Conceição das Alagoas y Monte Alegre de Minas.

Dichos municipios serán comparados con 10 municipios de tamaño medio en el mismo Estado: Chapecó, Juiz de Fora, São Leopoldo, Poços de Caldas, Petrópolis, Volta Redonda, Anápolis, Cubatão, Águas Lindas de Goiás y Lages.

Los 11 municipios pequeños se caracterizan por un Índice de Desarrollo Humano (IDH) significativamente inferior a la media brasileña y de los municipios de tamaño medio o de las grandes capitales. En estos 11 municipios, el IDH oscila entre 0,641 y 0,795 . En segundo lugar, todos poseen altas concen- 
Tabla 1.

Número de Consejos en municipios pequeños.

\begin{tabular}{lcccc}
\hline \multirow{2}{*}{ Ciudades } & \multicolumn{2}{c}{ Consejos } & Total \\
\cline { 2 - 5 } & Salud & Asistencia Social & $\begin{array}{c}\text { Infancia } \mathbf{y} \\
\text { Adolescencia }\end{array}$ & 3 \\
\hline Alpinópolis & 1 & 1 & 1 & 2 \\
\hline Alvinópolis & 1 & 0 & 1 & 3 \\
\hline Arinos & 1 & 1 & 1 & 3 \\
\hline Belo Oriente & 1 & 1 & 1 & 3 \\
\hline Bom Sucesso & 1 & 1 & 1 & 3 \\
\hline Campina Verde & 1 & 1 & 1 & 3 \\
\hline Carmo do Cajurú & 0 & 1 & 1 & 3 \\
\hline Carmo do Rio Claro & 1 & 1 & 0 & 1 \\
\hline Chapada do Norte & 1 & 1 & 1 & 3 \\
\hline Conceição das Alagoas & 1 & 0 & 10 & 29 \\
\hline Monte Alegre de Minas & 1 & 1 & & 2 \\
\hline Total & 10 & 9 & 1 & 3 \\
\hline
\end{tabular}

Fuente: Avritzer, 2016.

traciones de pobreza según el índice GINI, variando entre 0,38 y 0,59. Partiendo de esta caracterización bastante breve, es posible señalar que el fenómeno que continúa determinado, desde un punto de vista socioeconómico, la participación en estas pequeñas ciudades, es la pobreza y la falta de infraestructura. Así, el problema de la escala puede ser visto, de partida, desde otra óptica en el caso brasileño: el predominio de rentas bajas en los municipios de pequeño tamaño junto a prácticas políticas locales que favorecen el clientelismo y la dependencia política.

Nuestra investigación se centra en los Consejos de Salud, Asistencia Social e Infancia y Adolescencia, debido a la centralidad de dichos Consejos en el escenario de participación brasileño. Estas IP no solo tienen origen directo en la constitución de 1988, además, fueron las primeras en implantarse en las grandes capitales y a partir de ahí se generalizaron, mediante una estructura organizativa bastante semejante, en el nivel municipal brasileño (Avritzer 2009; Cunha 2009). En primer lugar, nos preguntamos por dicho diseño organizativo en los Consejos de las pequeñas ciudades para intentar analizar en qué medida reproducen esa configuración, dadas las deficiencias económicas, sociales y administrativas señaladas anteriormente. Los diseños para la participación en los Consejos se fueron homogeneizando a lo largo de la década de los 90. Estructuras inicialmente introducidas en las capitales, como las plenarias o las comisiones temáticas, se expandieron por las ciudades de tamaño medio según la dinámica temporal descrita en la Tabla 3.

Tabla 2.

Número de Consejos en municipios de tamaño medio.

\begin{tabular}{lcccc}
\hline \multirow{2}{*}{ Ciudades } & \multicolumn{2}{c}{ Consejos } & Total \\
\cline { 2 - 5 } & Salud & Asistencia Social & $\begin{array}{c}\text { Infancia y } \\
\text { Adolescencia }\end{array}$ & 3 \\
\hline Chapecó & 1 & 1 & 1 & 3 \\
\hline Juiz de Fora & 1 & 1 & 1 & 2 \\
\hline São Leopoldo & 0 & 1 & 1 & 3 \\
\hline Poços de Caldas & 1 & 1 & 1 & 3 \\
\hline Petrópolis & 1 & 1 & 1 & 3 \\
\hline Volta Redonda & 1 & 1 & 1 & 3 \\
\hline Anápolis & 1 & 1 & 1 & 3 \\
\hline Cubatão & 1 & 1 & 1 & 3 \\
\hline Águas Lindas de Goiás & 1 & 1 & 10 & 29 \\
\hline Lages & 1 & 1 & 1 & 3 \\
\hline Total & 9 & 10 & 1 & 3 \\
\hline
\end{tabular}

Fuente: Avritzer, 2016 
Tabla 3.

Año de creación del Reglamento Interno en vigor.

\begin{tabular}{|c|c|c|c|c|c|c|c|c|c|c|}
\hline \multirow{3}{*}{ Período } & \multicolumn{6}{|c|}{ Número de Consejos por período de creación del Reglamento Interno } & \multicolumn{4}{|c|}{ Total } \\
\hline & \multicolumn{2}{|c|}{ Salud } & \multicolumn{2}{|c|}{ Asistencia Social } & \multicolumn{2}{|c|}{ Infancia y Adolescencia } & \multicolumn{2}{|c|}{$\begin{array}{l}\text { Ciudades } \\
\text { medias }\end{array}$} & \multicolumn{2}{|c|}{$\begin{array}{l}\text { Ciudades } \\
\text { pequeñas }\end{array}$} \\
\hline & $\begin{array}{c}\text { Ciudades } \\
\text { Medias }\end{array}$ & $\begin{array}{l}\text { Ciudades } \\
\text { Pequeñas }\end{array}$ & $\begin{array}{l}\text { Ciudades } \\
\text { Medias }\end{array}$ & $\begin{array}{l}\text { Ciudades } \\
\text { Pequeñas }\end{array}$ & $\begin{array}{c}\text { Ciudades } \\
\text { Medias }\end{array}$ & $\begin{array}{l}\text { Ciudades } \\
\text { Pequeñas }\end{array}$ & $\mathbf{N}^{\circ}$ & $\%$ & $\mathbf{N}^{\circ}$ & $\%$ \\
\hline 1988-1995 & 0 & 1 & 0 & 0 & 0 & 0 & 0 & 0,0 & 1 & 3,45 \\
\hline $1996-2008$ & 5 & 3 & 8 & 4 & 7 & 4 & 20 & 69,0 & 11 & 37,93 \\
\hline 2009- en adelante & 0 & 3 & 0 & 3 & 0 & 4 & 0 & 0,0 & 10 & 34,48 \\
\hline Sin información & 4 & 3 & 2 & 2 & 3 & 2 & 9 & 31.0 & 7 & 24,14 \\
\hline Total & 9 & 10 & 10 & 9 & 10 & 10 & 29 & 100 & 29 & 100,0 \\
\hline
\end{tabular}

Fuente: Avritzer, 2016

Esta dinámica puede ser descrita de la siguiente forma: los Consejos en estas áreas surgieron en las capitales antes de establecerse nacionalmente, como en el caso del Consejo de Salud en la ciudad de São Paulo. Otros se crearon inmediatamente después de la aprobación de la legislación, como el Consejo de Salud de Belo Horizonte. Estos Consejos resultaron tremendamente influyentes a la hora de consolidar una institucionalidad participativa compuesta por los siguientes formatos: plenaria, mesa directora, secretaría ejecutiva y comisiones temáticas. Respecto a la dinámica de creación de los Consejos en ciudades pequeñas y medias, entre 1988 y 1995 solo se creó un Consejo en estas ciudades. Es decir, en ese momento, los Consejos se estaban creando en las capitales que contaban con un mayor poder de movilización de la sociedad civil. El periodo de formación de los Consejos en las ciudades de tamaño medio transcurre entre 1996 y 2008; en este lapso de tiempo, se creó el 69 \% de los Consejos en las ciudades medias de nuestra muestra. En las ciudades pequeñas, los Consejos se crearon, en parte, en dicho periodo y, en parte, (en un $34,48 \%$ de los casos) después de 2008. Así, desde el punto de vista temporal, hay una secuencia de creación de los Consejos determinada por el tamaño de las ciudades, secuencia que va a influir en la organización institucional.
Como se ha señalado anteriormente, la estructura organizativa de los Consejos, se constituyó durante los años 90, para influir a todas las ciudades brasileñas. En Avritzer (2010) se observa cómo los municipios de más de 100.000 habitantes reproducían esta estructura, lo que también sucede con las ciudades de tamaño medio ${ }^{3}$. Sin embargo, los datos recogidos en la Tabla 4 indican una enorme diferencia respecto a la presencia de dichas estructuras en las ciudades pequeñas y medianas. Todas las estructuras específicas están menos presentes en las ciudades pequeñas. Llama especialmente la atención cómo las estructuras que serían más participativas o que darían mayor calidad a la participación de la sociedad civil, como las comisiones temáticas o las conferencias municipales, son las que menor presencia tienen. Las comisiones temáticas están presentes en un $44,83 \%$ de los casos y las conferencias municipales solo en un $24,14 \%$, es decir, uno entre cuatro municipios. Podemos sugerir inicialmente, pese a que ampliaremos el análisis comparado de estos municipios, que los municipios de pequeño tamaño en Brasil presentan un déficit significativo en la estructura organizativa de la participación social cuando la comparamos con la misma estructura en municipios de tamaño medio.

Tabla 4.

Número de estructuras organizativas.

\begin{tabular}{llccc}
\hline \multirow{2}{*}{ Tipo de estructuras } & \multicolumn{3}{c}{ Número de Consejos } \\
\cline { 2 - 5 } & \multicolumn{2}{c}{ Ciudades medias } & \multicolumn{2}{c}{ Ciudades pequeñas } \\
\cline { 2 - 5 } & $\mathrm{N}^{0}$ & $\%$ & $\mathrm{~N}^{\circ}$ & 72,41 \\
\hline Plenaria & 25 & 86,21 & 21 & 55,17 \\
\hline Mesa Directora & 24 & 82,76 & 16 & 48,28 \\
\hline Secretaría Ejecutiva & 26 & 89,66 & 14 & 44,83 \\
\hline Comisiones Temáticas & 25 & 86,21 & 13 & 24,14 \\
\hline Conferencias Municipales & 22 & 75,86 & 7 & \\
\hline
\end{tabular}

Fuente: Avritzer, 2016 
Al análisis presentado hay que añadir una constatación adicional: mayor presencia del Gobierno y de sus prerrogativas exclusivas en los municipios de pequeño tamaño. La Tabla 4 tiene como objetivo sintetizar la presencia mayor o menor de prácticas democráticas en los diferentes Consejos a partir del tamaño de la ciudad. En relación a quién y cómo ocupa la presidencia de los Consejos fue posible inferir que, en el caso de los Consejos de Salud en las ciudades pequeñas, la presidencia es ocupada por el secretario municipal en apenas el $40 \%$ de los casos. Vale la pena mencionar que la ocupación de la presidencia por miembros de la sociedad civil es una bandera relativamente generalizada en el área de la salud, lo que hace que este dato sea especialmente significativo.

Según estos datos, una ciudad de tamaño medio tiene de dos a tres veces más posibilidades, dependiendo del Consejo en cuestión, de elegir un consejero cualquiera para la presidencia que una ciudad de tamaño pequeño. Observamos cómo se va afirmando un cierto patrón relativo a las prácticas democráticas dentro de los Consejos. Al mismo tiempo podemos observar, tal y como muestra la Tabla 6 , la diferencia entre los dos tipos de ciudades respecto a las reglas para la modificación de los reglamentos de los Consejos. En 18 de los Consejos en las ciudades de tamaño medio consideradas, las reglas exigen la aprobación del cambio de reglamento en una proporción predeterminada. En las ciudades pequeñas, la mayor parte no posee información, lo que probablemente significa que el reglamento no dice nada sobre el tema. De nuevo, existen motivos para pensar que los consejeros y la representación de la sociedad civil tienen más prerrogativas participativas en las ciudades de tamaño medio.
Podemos, por tanto, realizar algunas consideraciones parciales sobre la cuestión de la participación y la escala en las IP en Brasil. Mediante la comparación de las formas de participación en Consejos de pequeñas y medianas ciudades en el estado de Minas Gerais, es posible mostrar que las formas de participación son más avanzadas en las ciudades de tamaño medio. Tres elementos de sus diseños nos permiten confirmar este argumento: a) quién ocupa la presidencia en los Consejos de Salud, Asistencia Social e Infancia y Adolescencia; b) la autonomía del poder público para modificar el reglamento de los Consejos y; c) la presencia de conferencias municipales y comisiones temáticas. Estos elementos son fundamentales en la organización democrática de los Consejos y constituyen la evidencia de una forma más participativa de gestión centrada en la relación con la sociedad civil. Los datos presentados permiten apuntar deficiencias respecto a estas cuestiones y a otras; como la paridad de la sociedad civil en los Consejos donde también existen variaciones, aunque no tan significativas.

Una posible variable explicativa para esta cuestión está vinculada a la forma en la que se organiza el poder político en las pequeñas ciudades brasileñas. Es decir, la cuestión de la intensidad de las formas de participación en el nivel local en Brasil, aún parece fuertemente vinculada a las estructuras del poder local. Cuestiones como el control del poder político sobre la política municipal, estructuras sociales altamente jerarquizadas, y una sociedad civil incapaz de oponerse al sistema político, parecen ser los factores que más influencian la participación política en las pequeñas ciudades estudiadas en esta investigación. Estos datos contradicen las afirmaciones de algunos

Tabla 5.

Quién puede presidir el Consejo.

\begin{tabular}{|c|c|c|c|c|c|c|c|c|c|c|}
\hline \multirow{4}{*}{ Actor } & \multicolumn{6}{|c|}{ Número de Consejos según quién puede asumir la presidencia } & \multicolumn{4}{|c|}{ Total } \\
\hline & \multicolumn{2}{|c|}{ Salud } & \multicolumn{2}{|c|}{ Asistencia Social } & \multicolumn{2}{|c|}{$\begin{array}{c}\text { Infancia y } \\
\text { Adolescencia }\end{array}$} & \multicolumn{2}{|c|}{$\begin{array}{c}\text { Ciudades } \\
\text { medias }\end{array}$} & \multicolumn{2}{|c|}{$\begin{array}{l}\text { Ciudades } \\
\text { pequeñas }\end{array}$} \\
\hline & \multicolumn{6}{|c|}{ CIUDADES } & & & & \\
\hline & Medias & Pequeñas & Medias & Pequeñas & Medias & Pequeñas & $\mathbf{N}^{\circ}$ & $\%$ & $\mathbf{N}^{\circ}$ & $\%$ \\
\hline $\begin{array}{l}\text { Solo el secretario } \\
\text { municipal }\end{array}$ & 1 & 4 & 0 & 0 & 0 & 0 & 1 & 3,45 & 4 & 13,79 \\
\hline $\begin{array}{l}\text { Cualquier consejero } \\
\text { elegido en asamblea }\end{array}$ & 6 & 2 & 7 & 7 & 8 & 4 & 21 & 72,41 & 13 & 44,83 \\
\hline $\begin{array}{l}\text { Cualquier miembro } \\
\text { del Consejo elegi- } \\
\text { do con alternancia } \\
\text { bianual }\end{array}$ & 0 & 0 & 0 & 1 & 1 & 0 & 1 & 3,45 & 1 & 3,45 \\
\hline $\begin{array}{l}\text { Representante de la } \\
\text { Sociedad Civil pre- } \\
\text { sidente y vicepresi- } \\
\text { dencia del Gobierno }\end{array}$ & 0 & 0 & 0 & 0 & 0 & 1 & 0 & 0,00 & 1 & 3,45 \\
\hline Sin información & 2 & 4 & 3 & 1 & 1 & 5 & 6 & 20,69 & 10 & 34,48 \\
\hline Total & 9 & 10 & 10 & 9 & 10 & 10 & 29 & 100,0 & 29 & 100,0 \\
\hline
\end{tabular}

Fuente: Avritzer, 2016. 
Tabla 6.

Reglas de modificación del Reglamento Interno.

\begin{tabular}{|c|c|c|c|c|c|c|c|c|c|c|}
\hline \multirow{3}{*}{ Periodo } & \multicolumn{6}{|c|}{ Número de Consejos por modificación del Reglamento Interno } & \multicolumn{4}{|c|}{ Total } \\
\hline & \multicolumn{2}{|c|}{ Salud } & \multicolumn{2}{|c|}{ Asistencia Social } & \multicolumn{2}{|c|}{ Infancia y Adolescencia } & \multicolumn{2}{|c|}{$\begin{array}{c}\text { Ciudades } \\
\text { medias }\end{array}$} & \multicolumn{2}{|c|}{$\begin{array}{l}\text { Ciudades } \\
\text { pequeñas }\end{array}$} \\
\hline & $\begin{array}{c}\text { Ciudades } \\
\text { Medias }\end{array}$ & $\begin{array}{l}\text { Ciudades } \\
\text { Pequeñas }\end{array}$ & $\begin{array}{l}\text { Ciudades } \\
\text { Medias }\end{array}$ & $\begin{array}{l}\text { Ciudades } \\
\text { Pequeñas }\end{array}$ & $\begin{array}{c}\text { Ciudades } \\
\text { Medias }\end{array}$ & $\begin{array}{l}\text { Ciudades } \\
\text { Pequeñas }\end{array}$ & $\mathbf{N}^{\circ}$ & $\%$ & $\mathbf{N}^{\circ}$ & $\%$ \\
\hline $\begin{array}{l}\text { Consejeros } \\
\text { con proposi- } \\
\text { ción y apro- } \\
\text { bación }\end{array}$ & 0 & 2 & 0 & 0 & 2 & 1 & 2 & 6,90 & 3 & 10,34 \\
\hline $\begin{array}{l}\text { Consejeros } \\
\text { con propor- } \\
\text { ción de apro- } \\
\text { bación }\end{array}$ & 7 & 4 & 5 & 3 & 6 & 2 & 18 & 62,07 & 9 & 31,03 \\
\hline $\begin{array}{l}\text { Consejeros } \\
\text { sin criterios ni } \\
\text { proporción }\end{array}$ & 0 & 1 & 0 & 0 & 0 & 0 & 0 & 0,00 & 1 & 3,45 \\
\hline $\begin{array}{l}\text { Sin informa- } \\
\text { ción }\end{array}$ & 2 & 3 & 5 & 6 & 2 & 7 & 9 & 31,03 & 16 & 55,17 \\
\hline Total & 9 & 10 & 10 & 9 & 10 & 10 & 29 & 100,0 & 29 & 100,0 \\
\hline
\end{tabular}

Fuente: Avritzer, 2016

clásicos en el estudio de la democracia (Przeworski 2010; Dahl 1988). El caso de las ciudades de tamaño medio es interesante porque contradice presupuestos de la misma literatura, como la dificultad para la toma de decisiones y la coordinación de la participación en territorios más amplios. ¿Cómo debemos entender, entonces, la cuestión de la escala? En el próximo apartado de este artículo, ampliaremos las reflexiones sobre la relación entre participación y escala mediante el análisis de las Conferencias Nacionales.

\section{Conferencias Nacionales: conectando lo LOCAL CON LO NACIONAL}

Pese a que las Conferencias Nacionales aparecen en 1941, es a partir de 2003 con el comienzo del Gobierno nacional del Partido dos Trabalhadores cuando podemos afirmar que el impulso a las conferencias modifica el escenario participativo nacional (Avritzer y Souza 2013). Desde 1941 a finales del 2012 se celebran 125 conferencias nacionales, 90 entre 2003 y 2012, lo cual muestra claramente el impulso a estos procesos, con un carácter netamente participativo bajo dicho Gobierno. A este impulso le acompaña una importante ampliación de la agenda temática (Ramos y Faria 2013); de las 44 temáticas tratadas hasta el momento, 32 surgen tras 2003 (anteriormente estaban centradas en ámbitos específicos como la salud). A la hora de valorar el impacto cuantitativo de esta nueva institucionalidad, podemos señalar que el $6 \%$ de la población adulta de Brasil (unos 6 millones de personas), participó en alguna fase de las 74 Conferencias Nacionales que se dieron entre 2003 y 2010.
Las Conferencias presentan una importante capacidad de pautar la agenda nacional, especialmente en algunas áreas de política social. Por un lado, introduciendo importantes innovaciones como el SUAS (Sistema Único de Asistencia Social, fruto de la IV Conferencia Nacional de Asistencia Social), el Sistema Nacional de Seguridad Alimentaria, u otras decisiones en áreas como Políticas para Mujeres o Derechos Humanos. Pero también desde su capacidad de vetar algunas decisiones gubernamentales, como el bloqueo de la propuesta del Ministerio de Salud de introducir fundaciones nacionales, derrotada en dos Conferencias de Salud. De igual forma, en función del Estado, el municipio y/o la política pública, pueden surgir de las Conferencias Estatales o Municipales directrices para la elaboración de políticas públicas en dichos niveles, mostrando la capacidad del proceso para adaptarse a diferentes contextos administrativos. Por último, en estos procesos se ha demostrado una importante diversidad cognitiva que va más allá de los saberes de uso, respondiendo a otro de los interrogantes de la subida de escala (Ramos 2013).

Respecto al perfil de los participantes en las Conferencias, la participante típica es una mujer (51,2 \%), con cuatro años de escolaridad (26,9\%) o educación media completa $(20,3 \%)$ y una renta media entre 1 a 4 salarios mínimos (52,2 \%). Esto supone un patrón de participación similar al del nivel local, donde no son los más pobres los que participan, sino personas de renta media y, en general, con una escolaridad más alta que la media.

Junto al mantenimiento de la participación en áreas como Salud (la participación agregada es del 
$19,6 \%)$ o Asistencia Social $(36,4 \%)$, con una importante participación a nivel local tras la redemocratización, destaca el proceso de redefinición de intereses de los actores de la sociedad civil en Brasil, que muestra la importancia de ámbitos como Política para Mujeres (44,8\%) o Cultura $(29,4 \%)$.

Además del ciclo que va desde el nivel municipal hasta el cierre en el nivel nacional, el ciclo interno de cada fase es: a) plenaria de presentación, donde se lee el reglamento interno; b) división en grupos de trabajo (en adelante GT) para elaborar propuestas; c) elección de representantes y; d) plenaria de cierre para la aprobación definitiva de las propuestas.

A continuación exponemos algunas cuestiones relativas a las estructuras organizativas y a las prácticas de democratización relativas al diseño institucional de tres conferencias nacionales: Política para Mujeres (en adelante CNPM), Asistencia Social (en adelante CNAS) y Control Social y Transparencia (en adelante
CNCST). El análisis se centra fundamentalmente en elementos de su formato y en cómo evolucionan a medida que se sube de nivel. El objetivo es ver cuál de las escalas presenta una mayor calidad democrática. Para ello analizamos: a) presencia del reglamento interno (en adelante RI); b) democratización de la representación y; c) arquitectura deliberativa.

En primer lugar, observamos diferencias relativas a la organización y al número de participantes. La CNPM y la CNAS optaron por preconferencias municipales en los nueve distritos de Belo Horizonte (donde se eligen propuestas y delegados para la Conferencia Municipal). Por otro lado la CNCST recurrió a Conferencias Libres (son Conferencias que un grupo puede organizar cumpliendo con una serie de requisitos establecidos por la organización del proceso) y a la Conferencia Virtual (foro telemático de debate para la construcción de propuestas). El resultado de ambas (libres y virtual) se debatió en la fase nacional.

Tabla 7.

Si ya participó, en qué temática.

\begin{tabular}{llll}
\hline Temática & $\mathbf{N}^{\mathbf{0}}$ & $\%$ & Total \\
\hline Asistencia Social & 51 & 36,4 & 143 \\
\hline Comunicación & 16 & 11,2 & 143 \\
\hline Cultura & 42 & 29,4 & 143 \\
\hline Ciudades & 15 & 10,5 & 143 \\
\hline Derechos Humanos & 56 & 39,2 & 143 \\
\hline Educación & 27 & 18,9 & 143 \\
\hline Deportes & 23 & 16,1 & 143 \\
\hline Juventud & 16 & 11,2 & 143 \\
\hline Política para mujeres & 64 & 44,8 & 143 \\
\hline Salud & 12 & 8,4 & 143 \\
\hline Salud mental & 16 & 11,2 & 143 \\
\hline Seguridad alimentaria y nutricional & 5 & 3,5 & 143 \\
\hline Seguridad Pública & 18 & 12,6 & $143^{*}$ \\
\hline
\end{tabular}

Fuente: Avritzer, 2013. Tabla 3.

*Total de personas que afirman haber participado en las Conferencias Nacionales.

Tabla 8.

Número de participantes por temática y fase.

\begin{tabular}{|c|c|c|c|c|c|c|}
\hline \multirow[b]{2}{*}{ TEMÁTICA } & \multicolumn{6}{|c|}{ CONFERENCIAS } \\
\hline & Previas & $\begin{array}{l}\text { Municipal } \\
\text { de Belo } \\
\text { Horizonte }\end{array}$ & $\begin{array}{c}\text { Estatal de } \\
\text { Minas Gerais }\end{array}$ & $\begin{array}{l}\text { Nacional en } \\
\text { Brasilia }\end{array}$ & Libres & Virtual \\
\hline AS & $\begin{array}{c}9 \text { Conferencias } \\
\text { Distritales. } 2039 \\
\text { participantes }\end{array}$ & $\begin{array}{c}855 \\
\text { participantes }\end{array}$ & $\begin{array}{c}690 \\
\text { participantes }\end{array}$ & $\begin{array}{c}1820 \\
\text { participantes }\end{array}$ & No & No \\
\hline PPM & $\begin{array}{c}9 \text { Conferencias } \\
\text { Distritales. } 1092 \\
\text { participantes }\end{array}$ & $\begin{array}{c}317 \\
\text { participantes }\end{array}$ & $\begin{array}{c}957 \\
\text { participantes }\end{array}$ & $\begin{array}{c}2813 \\
\text { participantes }\end{array}$ & No & No \\
\hline CST & No & $\begin{array}{c}350 \\
\text { participantes }\end{array}$ & $\begin{array}{c}512 \\
\text { participantes }\end{array}$ & $\begin{array}{c}1374 \\
\text { participantes }\end{array}$ & $\begin{array}{l}\text { Si. } 16 \text { Conferencias } \\
\text { Libres en Minas } \\
\text { Gerais. La media } \\
\text { nacional fue de } 105 \\
\text { participantes. }\end{array}$ & $\begin{array}{l}\text { Si. } 19 \text { Marzo-8 de abril/ } \\
\text { 2012. } 2960 \text { participantes }\end{array}$ \\
\hline
\end{tabular}


El reglamento interno (en adelante $\mathrm{RI}$ ) de las Conferencias regula su funcionamiento en las diferentes fases y formaliza el proceso garantizando ciertos derechos a los participantes. La posibilidad de que dicho RI sea debatido en la Conferencia implica reconocer el derecho a debatir y alterar las condiciones de la deliberación y la representación.

Tanto la CNAS como la CNPM mejoran su institucionalidad y su democratización interna con la subida de escala al existir RI y posibilidad de debatirlo. En ambos casos el reglamento de la fase estatal era el más complejo al contemplar los criterios de deliberación y de representación/elección de delegados. En la CNCST pese a existir RI en las fases municipal y estatal, este no fue debatido, cosa que sí sucedió en la fase nacional.

La democratización de la representación está determinada por tres cuestiones: a) por la posibilidad de debatir y modificar sus características y reglas (recogidas en los RI) en las diferentes escalas; b) por la diversidad de criterios para la elección de delegados, y; c) por los mecanismos de elección. Respecto a la segunda cuestión, una mayor cantidad de criterios implica más diversidad de cuestiones para considerar la representación y esto, junto a la capacidad para deliberar sobre sus reglas, permite una mejor discusión sobre los proyectos políticos que se dan en torno de la representación. Entre los criterios existentes encontramos: la existencia de separación por segmentos (sociedad civil o poder público), cuotas para determinados colectivos o la atención a cuestiones territoriales (fundamentalmente el peso poblacional), y/o criterios de participación (que condicionan la cantidad de delegados a elegir). Respecto a los mecanismos de elección, existen mecanismos agregativos (donde se recurre a sistemas de elección más clásicos) o mecanismos más deliberativos (con posibilidad de discusión sobre la elección de delegados) bien para uno de los segmentos o para todos los segmentos posibles.

La CNCST mantiene los mismos criterios porcentuales de representación por segmento (60\% sociedad civil, $30 \%$ poder público y $10 \%$ consejeros) en todas las escalas. Es el proceso donde la elección es más claramente agregativa. Los participantes podían votar a los candidatos de su segmento mediante ur- nas electrónicas o votos en paneles. No siempre tenían tiempo para presentarse y cuando lo tenían era bastante reducido. La CNAS también diversifica los criterios de elección en la escala estatal, añadiendo a la separación por segmento (50 \% sociedad civil, $50 \%$ poder público) criterios poblacionales (número de habitantes por región). La elección solía darse en foros separados por segmento (con la diferencia de que los miembros del poder público no eran elegidos en la conferencia, sino previamente, limitando la publicidad del proceso). En dichos foros los candidatos se presentaban para que después se votase su candidatura. Esta votación estaba precedida de un debate sobre cómo desarrollar la propia elección ${ }^{4}$.

En la CNPM las condiciones de la representación pueden ser alteradas en todas las escalas en las que se discute el reglamento y se eligen delegados (municipal y estatal), y, a medida que se sube de nivel, más se complejiza la elección de delegadas (en la fase estatal). La combinación existente entre diversidad de criterios y posibilidad de discutir dichos criterios implica cambios importantes. En la fase municipal el porcentaje cambia de $60 \%$ sociedad civil y $40 \%$ poder público a $80 \%$ del primer segmento. Las modificaciones más importantes aparecen en la estatal, donde la representación se convierte en el elemento más polémico en los debates sobre el RI. Por un lado, cada una de las regionales ${ }^{5}$ consigue autonomía para elaborar sus pautas de elección, con lo que cada foro regional se convierte en un espacio de deliberación sobre cuáles son los criterios de representación y su legitimidad. Por otro lado, los pesos específicos de los criterios se alteran con la discusión y cambia la relación entre el criterio poblacional y el de participación (número de municipios que han realizado conferencias en cada división administrativa), pasando a tener más peso el segundo, con lo que cambia el porcentaje de delegadas a elegir por región.

Hay que destacar que mientras que la CNCST mantiene sus criterios de representación, la CNAS y la CNPM parten de una primera fase casi sin criterios (el número de delegados a elegir en las fases regionales dependía solo del número de asistentes y los foros eran mucho más informales) hacia una fase estatal donde se contemplaban numerosas variables para organizar la elección y la representación.

Tabla 9.

Existencia y posibilidad de votación del Reglamento Interno.

\begin{tabular}{|c|c|c|c|c|c|c|c|c|c|c|c|}
\hline \multirow{2}{*}{ Existencia de RI } & \multicolumn{3}{|c|}{ CNCST } & \multicolumn{4}{|c|}{ CNAS } & \multicolumn{4}{|c|}{ CNPM } \\
\hline & $\mathrm{CM}$ & CE & $\mathrm{CN}$ & PCM & $\mathrm{CM}$ & CE & $\mathrm{CN}$ & PCM & $\mathrm{CM}$ & CE & $\mathrm{CN}$ \\
\hline No existe RI & & & & $x$ & & & & $x$ & & & \\
\hline Existe RI, no se vota & $x$ & $x$ & & & & & & & & & \\
\hline Existe RI, se vota & & & $x$ & & $x$ & $x$ & $x$ & & $x$ & $x$ & $x$ \\
\hline
\end{tabular}

Fuente: Elaboración propia. 
Respecto a la arquitectura deliberativa, un ámbito de análisis se refiere a la metodología de trabajo y el tipo de debate que va a permitir. El primer elemento es la existencia o no de grupos de trabajo, pues su presencia supondría más oportunidades de participar en los debates temáticos. La segunda cuestión se refiere al porcentaje de propuestas que se someten a debate. El temario de las Conferencias suele estar estructurado en ejes temáticos y puede ocurrir que un GT trate menos de un eje, trate un eje completo, más de un eje o que se ocupe de todos los ejes señalados en el programa. Trabajar con menos de un eje implica una democratización menor de la discusión temática, al reducir el ámbito de participación de los actores, mientras que trabajar más de un eje permite una mayor transversalidad de la discusión. Por último hay que valorar la existencia de recursos formativos dentro del proceso, ya que son insumos que van a cualificar la discusión, además de ser un recurso para los propios participantes en sus ámbitos de trabajo o intervención respectivos más allá de la Conferencia.

Salvo en contadas excepciones (en algunas preconferencias de la CNPM) todas las conferencias contaron con GT, cuyo número aumentaba al crecer la cantidad de participantes con la subida de escala. Las diferencias se refieren al resto de elementos. En la CNCST, los GT se ocupaban de menos de un eje en algunas fases (estatal) y de solo un eje en otras (municipal y nacional). En las otras dos conferencias, el número de ejes tratados por los GT es mayor cuanto mayor es la escala; en la CNPM se trabajaba con dos ejes a partir de la estatal y en la CNAS donde se debatían todos los ejes en cada GT. Esta Conferencia fue la que hizo un esfuerzo mayor por mejorar la formación de los participantes, utilizando recursos diversos, entre los que destaca la presencia de grupos de teatro en las conferencias distritales. Las fases estatal y nacional eran las que más hincapié hacían en el aprendizaje a través de seminarios y talleres (especialmente la nacional). De forma similar, la CNPM dio más peso a la formación en las fases estatal y nacional, destacando esta última. La CNCST no considera importante dicho elemento dentro del proceso, limitándose a la realización de algunas ponencias en cada fase.

El segundo ámbito relativo a la arquitectura deliberativa se relaciona con la capacidad existente en cada una de las fases para que los GT puedan, bien modificar sustancialmente las propuestas, o bien, crear nuevas. Puede ser que los GT solo puedan seleccionar entre las propuestas procedentes de las fases anteriores, sin posibilidad de modificarlas (la escala con más legitimidad en este caso sería la municipal, al dar inicio al proceso y poder elaborar más las propuestas). Existiría otra posibilidad en la que los delegados tienen más autonomía para intervenir sobre los productos de las fases anteriores, rechazando, fusionando, modificando o creando propuestas nuevas. En este caso, se reconoce que la subida de escala genera un escenario de debate diferente, donde se discute sobre propuestas diferentes a las de la escala anterior y en torno a nuevos argumentos (los actores no son los mismos), y por tanto los delegados han de tener más capacidad para intervenir sobre la agenda.

En la CNPM, tanto en la fase estatal como en la nacional, los GT pueden modificar las propuestas derivadas de las fases anteriores y añadir propuestas nuevas. Esto supone un alto grado de modificación de las propuestas (alrededor del $90 \%$ en ambos casos). En el caso de la CNAS los GT de la fase estatal no pueden hacer propuestas nuevas, solo pueden modificar, mientras que sí pueden modificar y añadir nuevas en la nacional. Si bien no se alcanzan los niveles de la CNPM, los porcentajes de propuestas modificados en los dos niveles son muy elevados también. La CNCST limita el trabajo de los delegados estatales a la mera selección de propuestas y solo en la fase nacional podrán realizar algunas modificaciones, sin añadir propuestas nuevas.

Una polémica similar a la señalada con respecto a los GT existe con el rol que se otorga a la plenaria, que puede, o no, ser un espacio de deliberación, votación y/o construcción de propuestas. La importancia de este espacio se refiere a la consideración que se le da como un último espacio de elaboración del interés común y de ampliación de los actores que pueden dar su opinión sobre diferentes temáticas. Las diferencias estriban en qué posibilidades existen en este espacio.

De nuevo aparecen diferencias significativas entre las escalas y las temáticas. La CNCST optó en todos los niveles por el mismo formato. Los participantes votaban uno a uno las propuestas aprobadas en los grupos de trabajo. Es decir, tenían un número máximo de votos (10) para repartir entre las propuestas que consideraban mejores. La dinámica consistía en exponer las propuestas por escrito y que cada delegado votase en cada proyecto. La plenaria, por lo tanto, carecía de cualquier forma de discusión colectiva. Las CNPM y CNAS dan más valor a este espacio a la hora de construir intereses comunes y deliberaciones colectivas (presentando, discutiendo y votando las propuestas) y en los dos casos ese hecho mejora con la subida de la escala (las plenarias en la escala premunicipal son más informales). Dos elementos son importantes a la hora de considerar la calidad deliberativa de las plenarias. Solo en la fase estatal de la plenaria de mujeres se podían hacer propuestas nuevas, aunque en los dos casos y en las dos escalas las plenarias podían realizar modificaciones de lo deliberado en los grupos. Por otro lado, en la escala estatal la cantidad de propuestas que se somete a debate es mayor en ambos casos por dos razones. En primer lugar porque en este momento del ciclo se debaten 
propuestas para el nivel nacional y para el estatal, y no existen los filtros que existen en la nacional, donde se debate una cantidad predeterminada de propuestas que no han alcanzado la aprobación de un porcentaje establecido de los GT (aquellas con una aceptación del $70 \%$ de los GT no son sometidas a votación) en la estatal se debaten todas las propuestas que emergen de los GT.

Observamos dos tipos de diseño institucional. El primero no valoriza la escala y corresponde a la CNCST. En este ciclo, el RI solo se debate en la fase nacional, y los GT debaten sobre un porcentaje menor de las propuestas, con poca capacidad de modificación una vez que se supera el nivel municipal con lo cual no son capaces de valorizar los diferentes recursos cognitivos que aporta el scaling up. No se hace especial hincapié en la formación y las plenarias optan por mecanismos meramente agregativos en la selección de las propuestas finales. Si bien es cierto que cabe destacar el impulso a otras formas de participación como las Conferencias Libres y la Conferencia Virtual.

La CNPM y la CNAS serían ejemplos de diseños institucionales que sí valoran la escala. En ambos casos se pasa de un primer momento con escaso desarrollo institucional (la fase premunicipal) a una escala que supone una mejora de la calidad y de la capacidad democratizadora del proceso en varios elementos: a) mejora en la formación de los delegados; b) más atención a la democratización de la representación; c) incremento de la calidad deliberativa de las mismas; d) aumento de la transversalidad del proceso, al deliberar sobre un porcentaje mayor de los ejes temáticos y; e) mayor complejidad en los reglamentos internos de las conferencias. Además, ambos procesos cuentan con propuestas para los diferentes niveles de gobierno, algo que no sucede con la CNCST, limitada a la esfera nacional.

En los tres casos, los diseños institucionales y su relación con la escala dependen de qué tipo de políticas públicas son (Mujeres y Asistencia tienen un desarrollo como arena participativa mucho mayor) y del grado de desarrollo de la sociedad civil en cada caso. Pese a que la corrupción y la transparencia se han convertido en una agenda fundamental en la actualidad en Brasil, en torno a la política para Mujeres y de Asistencia Social y en las escalas estatal y nacional, la movilización social estaba más desarrollada y más diversificada (con respecto a las escalas municipales).

\section{Conclusiones: PARTICIPACIÓn EN BRAsil, REEVALUANDO LA CUESTIÓN DE LA ESCALA}

La hipótesis de partida de este trabajo era que, en oposición al consenso de gran parte de la teoría democrática que afirma que son las escalas menores las que presentan mejores condiciones participativas, existen evidencias empíricas en el panorama participativo brasileño que señalan que serán los niveles intermedios (escala estatal y municipios de tamaño medio) los que presentan unas mejores condiciones. La metodología desarrollada nos ha permitido abordar esta hipótesis que vincula la eficacia de la participación con el tamaño del territorio y el nivel administrativo, comparando dos tipos de Instituciones Participativas. Por un lado, los Consejos de salud, asistencia social y derechos de la infancia y la adolescencia en municipios de diferente tamaño. Por otro lado, las Conferencias Nacionales, de mujeres, asistencia social y transparencia, en diferentes niveles administrativos: municipal, estatal y nacional.

Los resultados mostrados en estas páginas corroboran nuestra hipótesis de partida: son los niveles intermedios los que presentan mejores condiciones y características participativas, una constatación que además nos permite analizar dos elementos relacionados con el vínculo entre participación y escala: el narrowing down y el scaling up.

Al analizar la misma tipología de IP en municipios con diferente tamaño podemos mostrar las dimensiones fundamentales del fenómeno del narrowing down en ciudades pequeñas, que están lejos de ser las que presentan las mejores condiciones para la participación. La primera alude a un desarrollo institucional más precario de la institucionalidad participativa en los municipios pequeños en comparación con los municipios de tamaño intermedio. Esta cuestión se refiere a: a) la ausencia en los municipios pequeños de las estructuras más participativas (mesas temáticas y/o conferencias municipales) que mejorarían la inclusión en estas IP; b) la mayor presencia y poder de los gobiernos locales en dichos Consejos, en detrimento de la sociedad civil, y; c) ausencia de reglas claras que aseguren un marco democrático de interacción poder público-sociedad civil en dichos municipios. Este déficit de la estructura organizativa de la participación social está ligado a una institucionalización más tardía de estos procedimientos.

La segunda dimensión del narrowing down tiene que ver con la cultura política y, en estos casos, también responde al hecho de que el nivel local en Brasil presenta aún una continuidad significativa por prácticas clientelistas y de dependencia política (Chilcote 1982; Leal 1975; Avritzer 2010). En el caso de las ciudades de tamaño medio los datos señalan un fuerte esfuerzo democrático. Un esfuerzo probablemente ligado a una organización más independiente de la sociedad civil y a la presencia de estructuras sociales menos dependientes del poder político.

En el caso de las Conferencias Nacionales ${ }^{6}$, en aquellos diseños que valorizan la escala (política para Mujeres y Asistencia Social), el nivel estatal es el más democrático. Por un lado, es cierto que sus estructuras deliberativas (aunque con diferencias) son simila- 
res en las escalas superiores, y que el nivel nacional presenta un esfuerzo mayor en la formación de los participantes. Por otro lado, ambos niveles presentan una mejor adecuación entre el diseño participativo de producción de demandas y la escala o nivel administrativo que ha de responder al mismo; es decir, de su impacto en la redacción de planes de políticas públicas apropiados para cada escala. Pero a la hora de determinar cuál es la escala que presenta una mayor calidad, son varios los elementos que nos llevan a afirmar que es la fase estatal: a) en ambos casos los porcentajes de transversalidad en los ejes temáticos son altos, pero las escalas estatales aumentan los contenidos de la deliberación al dirigirse a dos niveles (nacional y estatal); b) Las plenarias tienen características más democráticas en la fase estatal. Comenzando porque en el caso de la CNPM dicho foro tiene la capacidad de hacer propuestas nuevas en dicho nivel, cosa que no sucede en la CNAS. En ambas fases (estatal y nacional) la plenaria puede modificar las propuestas que lleguen de los GT, sin embargo en el último nivel del proceso las propuestas sobre las que se delibera son porcentualmente menores. En el Estado, la plenaria delibera sobre todos los proyectos que surgen de los GT, en la fase nacional solo se debaten aquellos que no han alcanzado un porcentaje determinado de aprobación previa. Esto permite un debate ampliado en el que otros actores pueden señalar opiniones diferentes respeto a lo aprobado y pueden dirimirse conflictos internos entre los participantes que, por la composición de los GT no se han discutido; y; c) la representación es otra de las cuestiones diferenciales. Los ámbitos más conflictivos dentro del proceso conferencista son la priorización de propuestas y la elección de delegados para la fase siguiente. Esta última cuestión no aparece en la fase nacional y junto a lo anterior corrobora que ante condiciones democráticas similares, la fase estatal aborda un conjunto mayor de cuestiones que la fase nacional. Es en el nivel del Estado donde la representación presenta características más diversificadas, destacando además las posibilidades que se dan en el proceso de la CNPM de intervenir sobre las reglas.

El análisis de las Conferencias Nacionales nos permite superar las perspectivas débil y fuerte del scaling up mostradas anteriormente. Por un lado mostrando la importancia de su arquitectura institucional y la necesaria interacción entre foros y es- pacios, así como mostrando las potencialidades de las instituciones híbridas y de arquitecturas participativas que van más allá de los minipúblicos a la hora de responder a las preguntas sobre las capacidades ciudadanas, la consolidación de los procesos, su inclusividad o el impacto de los mismos. Las Conferencias Nacionales muestran cómo el problema de la transmisión de las demandas locales hacia arriba encuentra solución mediante la realización de encuentros intermedios con una serie de requisitos institucionales, así como de elementos que aseguran su calidad deliberativa y mediante los cuales las cuestiones discutidas a nivel local vuelven a ser discutidas y complementadas con otras perspectivas que mejoran su correspondencia con la escala administrativa que tiene que dar respuesta a las mismas. Asimismo, en los casos estudiados existen formas de relación entre participación y representación que son diferentes de los dispositivos clásicos de la deliberación y que van a plantear problemas diferentes a los que señalan el giro sistémico y el scaling up respecto a la relación entre representación, participación/deliberación y escala.

El estudio de Consejos y Conferencias Nacionales desarrollado en este trabajo señala la posibilidad de implementar instituciones participativas relacionadas con las mismas políticas públicas en escalas diferentes. La primera conclusión de esta afirmación implica que la participación puede desarrollarse en territorios de gran extensión o con cantidades altas de habitantes o en niveles administrativos como el nacional. Abordando además agendas de alta complejidad (lejos de las necesidades inmediatas o los problemas de proximidad), bien en territorios concretos de diferentes tamaños o mediante la interacción entre foros diferentes en diferentes localidades y niveles administrativos distintos. La segunda es que lejos de entender la escala como una variable explicativa o determinante por sí misma de la calidad democrática de los procesos, es necesario prestar atención a las potencialidades democratizantes de los diseños institucionales y a sus relaciones con la cultura política y las características de la sociedad civil. Este vínculo entre cultura política, sociedad civil y diseños institucionales (en muchos casos resultantes de la interacción entre las dos primeras variables), explican que en las experiencias analizadas, las escalas más democráticas sean las intermedias.

\section{Notas}

1. Los municipios pequeños tienen menos de 20.000 habitantes, los medios más de 100.000 y las grandes ciudades más de 500.000 .

2. La encuesta se realizó en colaboración con el Instituto Vox Populi. Se seleccionó una muestra de 2200 personas, representativa de todo el país y que refleja la estratificación de renta, escolaridad, género y raza de Brasil.
3. Del total de Consejos analizados en 39 ciudades (tamaño medio, grande y grandes capitales), el 95,12 $\%$ tiene plenarias y el $82,11 \%$ comisiones temáticas (Avritzer 2010).

4. Nos referimos en este caso al método concreto de elección, ya que el porcentaje de delegados por segmento y territorio estaba predeterminado. 
5. La elección de delegadas se daba en cada una de las 20 divisiones regionales en las que se dividía el Estado de Minas Gerais.

6. No estudiamos en este caso el narrowing down en el proceso de Conferencias, al comparar tres escalas administrativas y no municipios de diferente tamaño. Sin

\section{Referencias Bibliográficas}

Avritzer, L. 2009. Participatory institutions in democratic Brazil. Washington: Wilson Press/Johns Hopkins University Press.

Avritzer, L. 2013. "Conferências Nacionais: ampliando e redefinindo os padrões de participação social no Brasil". Pp. 125-140 en Conferências nacionais: atores, dinámicas participativas e efetividade, coordinado por $\mathrm{L}$. Avritzer y C. Souza. Brasilia: IPEA.

Avritzer, L. 2016. "Participação em pequenas cidades no estado de Minas Gerais". Relatorio de pesquisa. FAPEMIG, Universidad Federal de Minas Gerais. Belo Horizonte, BR

Avritzer, L. 2010. A dinâmica da participação local no Brasil. São Paulo: Cortez.

Avritzer, L. y C. Sousa. 2013. Conferências nacionais: atores, dinámicas participativas e efetividade. Brasilia: IPEA.

Bächtiger, A. y A. Wegmann. 2014. "Scaling Up" Deliberation". Pp. 118-135 en Deliberative Democracy. Issues and Cases, coordinado por S. Elstub y P. McLaverty. Edinburgh: Edinburgh University Press.

Barber, B. 1984. Strong Democracy. Berkeley: University of California Press.

Bobbio, N. 1987. The future of democracy. Mineapolis: University of Minesota Press.

Chilcote, R. H. 1982. Partido Comunista Brasileiro: conflito e integração. Rio de Janeiro: Graal.

Cohen, J. 1997. "Procedure and Substance in Deliberative Democracy". Pp. 407-437 en Deliberative Democracy, coordinado por J. Bohman y W. Rehg. Cambridge: MIT Press.

Cunha, E. 2009. "Efetividade deliberativa: estudo comparado de Conselhos municipais de assistência social (1997-2996)". Ph.D. dissertation. Departamento de Ciencia Política, Universidad Federal de Minas Gerais. Belo Horizonte, BR.

Dahl, R. 1988. On Democracy. New Haven: Yale University Press.

Dahl, R. y E. Tufte. 1973. Size and Democracy. Stanford: Stanford University Press.

Dryzek, J. 2000. Deliberative Democracy and Beyond. Oxford: Oxford University Press.

Sartori, G. 1987. The theory of democracy revisited. Chatham, N. J.: Chatham House Publishers.

Faria, C. 2005. "O estado em movimento: complexidade social e participação politica no Rio Grande do Sul". Ph.D. dissertation. Departamento de Ciencia Política, Universidad Federal de Minas Gerais. Belo Horizonte, BR.

Friedman, W. 2006. "Deliberative Democracy and the problem of scope". Journal of Public Deliberation 2:1. Consultado 13 de noviembre del 2013 (http://www. publicdeliberation.net/jpd/vol2/iss1/art1/).

Fung A., E. O. Wright. 2003. Deeping Democracy. Institutional Innovations in Empowered Participatory Governance. London: Verso.

Habermas, J. 1995. Between facts and norms. Cambridge: MIT Press.

Hamilton A., J. Jay y J. Madison. 1988. Le Fédéraliste. Paris: Nouveaux Horizons. embargo las evidencias de nuestra investigación podrían confirmar dicha tendencia, ya que en los municipios pequeños del estado de Minas Gerais analizados, para otros fines, se muestra un escaso desarrollo institucional similar al de los Consejos aquí mencionados.

Hendriks C. 2006. "Integrated deliberation: Civil Society's dual role in deliberative democracy". Political Studies 54: 486508. http://dx.doi.org/10.1111/j.1467-9248.2006.00612.x

Jacobs, L., Cook, F. L. y M. X. Delli Carpini. 2009. Talking together: Public Deliberation and Political Participation in America. Chicago: University of Chicago Press. http:// dx.doi.org/10.7208/chicago/9780226389899.001.0001

Leal, V. N. 1975. Coronelismo, enxada e voto. São Paulo: Alfa Omega.

Levine, P., A. Fung y J. Gastill. 2005. "Future directions for Public Deliberation". Journal of Public Deliberation 1:1. Consultado 13 de noviembre del 2013 (http://www. publicdeliberation.net/jpd/vol1/iss1/art3/).

MacPherson C. 1977. The Life and Times of Liberal Democracy. Oxford: Oxford University Press.

Mansbridge J. y J. Parkinson. 2012. Deliberative Systems. Cambridge: Cambridge University Press.

Montesquieu B. 1951. L'espirit des lois. Paris: Gallimard.

Pateman, C. 1980. Participation and Democratic Theory. Cambridge: Cambridge University Press.

Pires, R. 2011. Efetividade das Instituições Participativas no Brasil: Estratégias de avaliação. Brasilia: IPEA.

Pogrebinschi T. y F. Santos. 2011. "Participação como Representação: O Impacto das Conferências Nacionais de Políticas Públicas no Congresso Nacional". DADOS 54:259-305.

Przeworski, A. 2010. Democracy and the Limits of SelfGovernment. New York: Cambridge University Press. http://dx.doi.org/10.1017/CBO9780511778490

Ramos, A. 2013. "Conferência Nacional de Política para as Mulheres: interações discursivas e implicações para a legitimidade epistêmica". Pp. 95-124 en Conferências nacionais: atores, dinámicas participativas e efetividade, coordinado por L. Avritzer y C. Souza. Brasilia: IPEA.

Ramos A. y C. Faria. 2013. "Las Conferencias de Políticas Públicas en Brasil". Revista Española de Ciencia Política 32:43-63.

Rousseau J.-J. 1992. Du Contrat Social. Paris : Classiques de la Philosophie.

Sintomer Y. 2008. "Du savoir d'usage au métier de citoyen?". Raisons Politiques 31:115-133. http://dx.doi. org/10.3917/rai.031.0115

Sintomer Y. y J. Talpin. 2011. "Introduction. Équiper ou dépasser la proximité?". Pp. 11-25 en La démocratie participative au-delà de la proximité, coordinado por Y. Sintomer y J. Talpin. Rennes: Presses Universitaries de Rennes.

Smith, G. 2009. Democratic Innovations: Designing institutions for citizen participation. Cambridge: Cambridge University Press. http://dx.doi.org/10.1017/ CBO9780511609848

Wampler, B. 2008. Participatory Budgeting in Brazil: Contestation, cooperation and accountability. University Park: Pennsylvania State Press.

Weber, M. 1978. Economy and Society. Berkley: University of California Press. 
LEONARDO AVRITZER es profesor de Ciencia Política en la Universidad Federal de Minas Gerais y director del Proyecto Democracia Participativa en la misma Universidad y presidente de la Asociación Brasileña de Ciencia Política. Es autor, entre otros, de Democracy and the Public Space in Latin America, Participatory Institutions in Democratic Brazil e Impasses da Democracia no Brasil.
ALFREDO RAMOS es doctorando en Ciencia Política en la Universidad Complutense de Madrid. Sus áreas de interés son la teoría democrática y la democracia participativa. Ha publicado artículos en revistas como Encrucijadas o la Revista Española de Ciencia Política. 